\title{
Le TGV Rhin-Rhône dans tous ses états
}

The French Rhine-Rhone HSR line: statement

Der TGV Rhin-Rhône im Überblick

\section{Raymond Woessner}

\section{(2) OpenEdition}

\section{Journals}

Electronic version

URL: http://journals.openedition.org/rge/5497

ISSN: 2108-6478

\section{Publisher}

Association des géographes de l'Est

\section{Printed version}

Date of publication: 1 October 2015

ISSN: 0035-3213

\section{Electronic reference}

Raymond Woessner, "Le TGV Rhin-Rhône dans tous ses états », Revue Géographique de l'Est [Online], vol.55 / n³-4 | 2015, Online since 11 October 2015, connection on 08 September 2020. URL : http:// journals.openedition.org/rge/5497

This text was automatically generated on 8 September 2020

Tous droits réservés 


\title{
Le TGV Rhin-Rhône dans tous ses états
}

\author{
The French Rhine-Rhone HSR line: statement \\ Der TGV Rhin-Rhône im Überblick
}

\section{Raymond Woessner}

1 Depuis le 11 décembre 2011, la France dispose d'une cinquième Ligne à Grande Vitesse (LGV), venue après celles du Sud-Est (1981), de l'Ouest (1989), du Nord (1993) et de l'Est (2007). Dijon et Besançon étaient déjà desservies par le TGV Sud-Est depuis 1981, la Lorraine et l'Alsace par le TGV-Est depuis 2007 et même par le TGV Méditerranée depuis 2004. Établie sur $140 \mathrm{~km}$ entre la Bourgogne et l'Alsace via la Trouée de Belfort, la LGV Rhin-Rhône est toutefois différente de ce qui a été réalisé précédemment (Figure 1). Elle apporte une autre dimension bien que ce TGV reste un peu mystérieux dans son essence même. Il semble aller et venir de partout entre Paris, Strasbourg, Zurich et Lyon, sans compter ses extensions vers le sud comme vers le nord. Le TGV Rhin-Rhône restera-t-il d'abord français ou sera-t-il un outil européen ? Pourra-t-il ramener de la croissance économique et démographique vers des territoires malmenés par la métropolisation et la désindustrialisation ? Quelles actions publiques et privées faut-il mettre en œuvre pour créer un effet TGV ? La Métropole Rhin-Rhône va-t-elle arracher sa région à elle-même ? Les questions se bousculent pour des villes qui se cherchent un destin. Et les territoires qui craignent de sortir du jeu voient une opportunité pour se reconnecter à la croissance ; mais que peuvent-ils entreprendre?

2 Face à la complexité des problèmes, du jeu des acteurs et de l'enchevêtrement des échelles géographiques, on se posera huit questions pratiques pour essayer de comprendre ce qui vient de se produire et ce qui se noue désormais :

1. La LGV Rhin-Rhône est-elle le produit d'un lobbying local ?

2. L'objectif de l'Association Trans Europe Rhin-Rhône Méditerranée est-il atteint?

3. Le TGV Rhin-Rhône est-il nécessaire?

4. Quels sont les périls menaçant l'achèvement de la LGV ?

5. Avec un projet comportant deux gares, les Dijonnais sont-ils dans l'erreur? 
6. La gare de Mulhouse est-elle un pôle de croissance?

7. Le syndrome des betteraves menace-t-il les gares nouvelles?

8. La Métropole Rhin-Rhône est-il un concept mort-né ?

Figure 1 : La LGV Rhin-Rhône et les autres TGV du Val-de-Saône, de la Franche-Comté et du SudAlsace

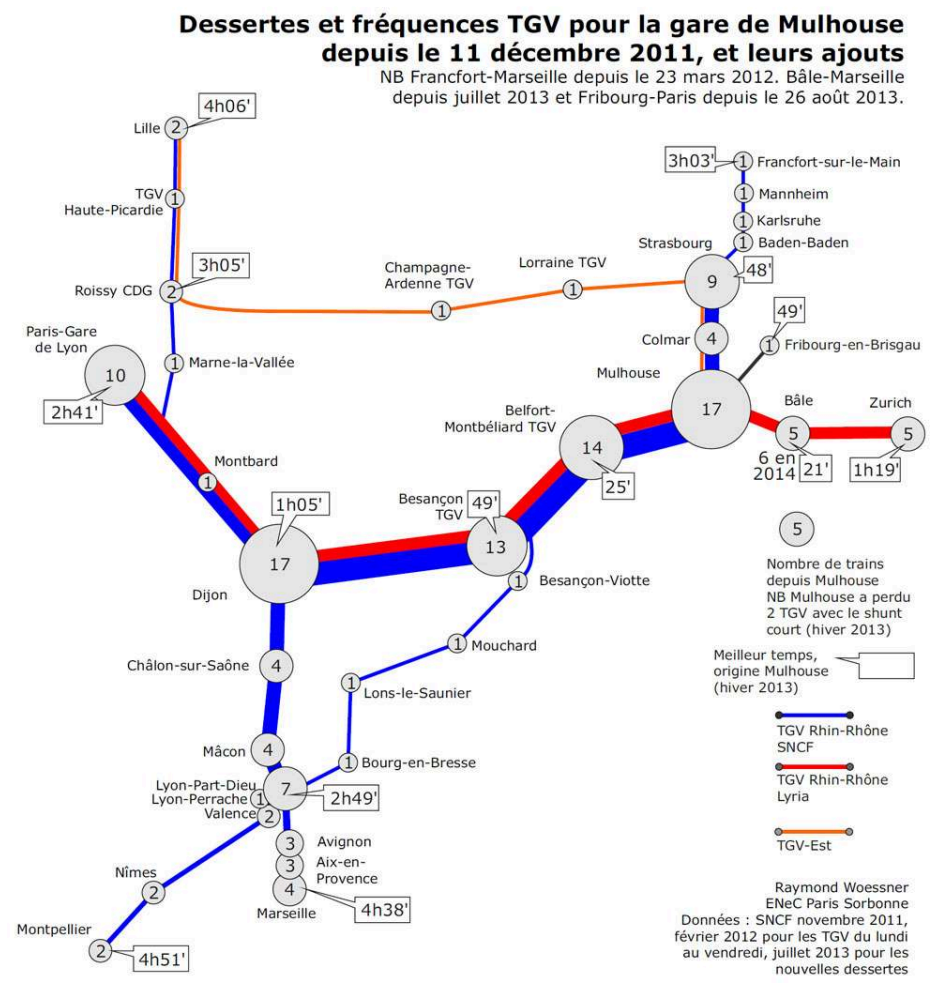

\section{La LGV Rhin-Rhône est-elle le produit d'un lobbying local ?}

3 Les premières LGV françaises avaient été décidées autour de la SNCF, d'Alsthom ${ }^{1}$, du ministère des Transports, de celui des Finances, avec des arbitrages rendus au sommet de l'Etat. Puis une évolution notable s'est produite avec le projet de la LGV-Est lorsque les collectivités ont fait pression pour sa concrétisation, avec un prix à payer, celui de leur participation financière.

4 L'initiative du projet de TGV Rhin-Rhône a été belfortaine (Woessner, 2000 ; 2005). Au début des années 1980, M. Chevènement est aux affaires en tant que ministre et députémaire de Belfort. Il fait alors feu de tout bois. Il lance la création de l'Université de Technologie de Belfort-Montbéliard (UTBM) malgré les réticences des Alsaciens et des Bisontins. Il obtient la création de l'Aire urbaine 2000 qui regroupe deux villes rivales, d'une part Belfort avec Alsthom, ses casernes, ses missiles nucléaires Pluton, sa préfecture, sa bourgeoisie catholique, et d'autre part Montbéliard forte de Peugeot et de sa haute société protestante.

5 En 1986, lorsque le rapport de l'inspecteur général Rattier pointe le risque de marginalisation de la Franche-Comté par la grande vitesse ferroviaire, $M$. Chevènement met sur pied l'Association Trans-Europe TGV, devenue depuis TGV Trans-Europe- 
Rhône-Méditerranée et tenue jusqu'en 2011 par son secrétaire M. Thierry Zettel. Cette association aura inlassablement labouré le terrain pendant plus de vingt ans. Dès 1988, elle propose la structure en $Y$ du réseau Rhin-Rhône entre l'Alsace, l'Ile-de-France et Rhône-Alpes. Au début des années 1990, le projet est inscrit dans les schémas directeurs français et européens ; dans la foulée, les premières études sont engagées par la SNCF. En novembre 1997, le colloque de Mulhouse réunit la fine fleur des responsables politiques du Rhin et de la Saône quelques jours après l'abandon du projet de canal Rhin-Rhône à grand gabarit ; l'on se promet alors la « concomitance » des travaux de la LGV-Est et de la LGV Rhin-Rhône, mais finalement le premier gardera quatre années d'avance sur le second. Le 25 janvier 2002, l'Etat publie la Déclaration d'Utilité Publique pour une LGV entre Dijon et Mulhouse. En 2003, la Commission européenne inscrit les branches Est et Sud dans les liaisons d'intérêt communautaire, ce qui ouvre la possibilité d'une contribution financière de sa part, devenue effective à hauteur de 8,65\%. En 2006, Les Magny (Haute-Saône) voit démarrer le chantier de la LGV. Au même moment, la Métropole Rhin-Rhône fédère la plupart des villes concernées par la LGV, et même au-delà.

6 Il a donc fallu une génération pour faire aboutir une partie de la LGV Rhin-Rhône. Le projet revient de loin. La SNCF s'était intéressée tardivement au Grand Est français. Même pour le TGV-Est, les perspectives de marché lui semblaient médiocres. Il est vrai que les villes françaises à desservir sont plus modestes que ne le sont Lille, Lyon ou Marseille, voire les villes de l'Atlantique où le TGV éclate sur plusieurs branches dès qu'il sort du Bassin parisien. Mais c'était sans compter avec les régions rhénanes de l'Allemagne et de la Suisse qui apportent environ $25 \%$ de la clientèle du TGV-Est. Les Strasbourgeois touchent à présent les dividendes du concept de la Magistrale Esteuropéenne Paris-Munich, en attendant de pouvoir aller jusqu'à Vienne voire Bratislava et Budapest, et en se réjouissant de la mise en chantier des $117 \mathrm{~km}$ de nouvelles lignes à grande vitesse du projet Stuttgart 21 (Bade-Wurtemberg).

7 Entre Rhône et Rhin, les perspectives commerciales sont encore plus compliquées, même en mettant Bâle et Zurich dans le jeu. On n'a cessé de perdre des liaisons sur la ligne $\mathrm{N}^{\circ} 4$ de la SNCF : le TEE Arbalète Zurich-Paris en 1979, le Bâle-Paris en 2007, le Mulhouse-Paris en 2011...

8 Les deux LGV de l'Est français se sont inscrites dans une démarche originale où l'argent public assurait le financement grâce à la contribution du mille-feuille administratif. Il en résulte un coût au kilomètre qui reste raisonnable (Figure 2), avec 16,51 millions d'euros au km pour la LGV Rhin-Rhône (140 km de ligne nouvelle). A l'avenir, les montages vont sans doute relever de Partenariats Publics Privés (PPP) à l'image de celui conclu pour la LGV Atlantique entre Tours et Bordeaux par la société Lisea et l'Etat. Selon la manière de calculer, ce PPP $(7,8$ milliards pour $340 \mathrm{~km})$ est de $38 \%$ à $56 \%$ plus cher au kilomètre que Rhin-Rhône : 22,94 millions, voire 25,88 millions si l'on inclut les travaux programmés par RFF pour la modernisation des accès à la future LGV. Il est vrai qu'entre-temps, la sécurisation environnementale a fait grimper le coût des travaux en général, en particulier avec la promulgation de la loi sur l'eau en décembre 2006. 
Figure 2 : Le coût de la branche Est Villers-les-Pots (Côte-d'Or) - Petit-Croix (Territoire de Belfort) en millions d'euros

\begin{tabular}{|c|c|c|c|c|c|}
\hline Alsace & 206 & $8,90 \%$ & Conseil régional & 104,7 & $4,50 \%$ \\
\hline & & & Bas-Rhin & 12,875 & $0,56 \%$ \\
\hline & & & Haut-Rhin & 53,175 & $2,30 \%$ \\
\hline & & & Strasbourg & 12,875 & $0,56 \%$ \\
\hline & & & Mulhouse & 15,175 & $0,66 \%$ \\
\hline & & & Colmar & 6,95 & $0,30 \%$ \\
\hline Franche-Comté & 316 & $13,70 \%$ & Conseil régional & 189,6 & $8,20 \%$ \\
\hline & & & Doubs & 39 & $1,69 \%$ \\
\hline & & & Territoire de Belfort & 25,5 & $1,10 \%$ \\
\hline & & & Haute-Saône & 17,1 & $0,74 \%$ \\
\hline & & & Jura & 13,3 & $0,58 \%$ \\
\hline & & & Besançon & 13,2 & $0,57 \%$ \\
\hline & & & Montbéliard & 9,217 & $0,40 \%$ \\
\hline & & & Belfort & 9,217 & $0,40 \%$ \\
\hline Bourgogne & 131 & $5,70 \%$ & Conseil régional & 68,1 & $2,90 \%$ \\
\hline & & & Côte-d'Or & 31,4 & $1,36 \%$ \\
\hline & & & Dijon & 31,4 & $1,36 \%$ \\
\hline Collectivités locales & 653 & $28,30 \%$ & \multirow{5}{*}{\multicolumn{3}{|c|}{$\begin{array}{r}\text { Données : Association TGV Rhin- } \\
\text { Rhône Méditerranée, } 2007\end{array}$}} \\
\hline Etat & 751 & $32,50 \%$ & & & \\
\hline RFF & 642 & $27,65 \%$ & & & \\
\hline Suisse & 66 & $2,90 \%$ & & & \\
\hline Union européenne & 200 & $8,65 \%$ & & & \\
\hline
\end{tabular}

NB : les villes mentionnées ont utilisé des moyens de financement variés en fonction de l'état de leurs structures intercommunales.

9 Au coût de l'infrastructure s'ajoute celui du matériel roulant. La SNCF affirme avoir acheté 30 rames Duplex tricourant de 509 sièges dont le prix catalogue est d'environ 30 millions d'euros l'unité. Techniquement, ces rames peuvent circuler en France, en Allemagne, en Suisse et au Luxembourg. La SNCF rénove également d'autres matériels pour les affecter progressivement à la ligne Rhin-Rhône. De son côté, la société Lyria, détenue à $74 \%$ par la SNCF et à $26 \%$ par les Chemins de Fer Fédéraux (CFF) suisses, est engagée dans un important programme de renouvellement de son matériel qui date principalement de 1981. Elle utilisera également des Duplex.

\section{L'objectif de l'Association Trans Europe Rhin-Rhône Méditerranée est-il atteint?}

Pour relancer la machine, l'idée de génie de l'Association Trans-Europe TGVMéditerranée a été de promouvoir une LGV européenne entre le Rhin supérieur jusqu'à l'Espagne via la vallée du Rhône. Ainsi, sur le croquis marketing de son site internet ${ }^{2}$, Paris n'était même pas connecté par une flèche (Document 1)! On est à la limite de la provocation quand on sait que les LGV françaises reproduisent fidèlement « l'étoile de Legrand », qui a fait rayonner les grandes lignes ferroviaires depuis Paris en 1842. Force est de constater que l'audace a payé... 
Document 1 : Présentation du projet par l'Association TGV Rhin-Rhône Méditerranée

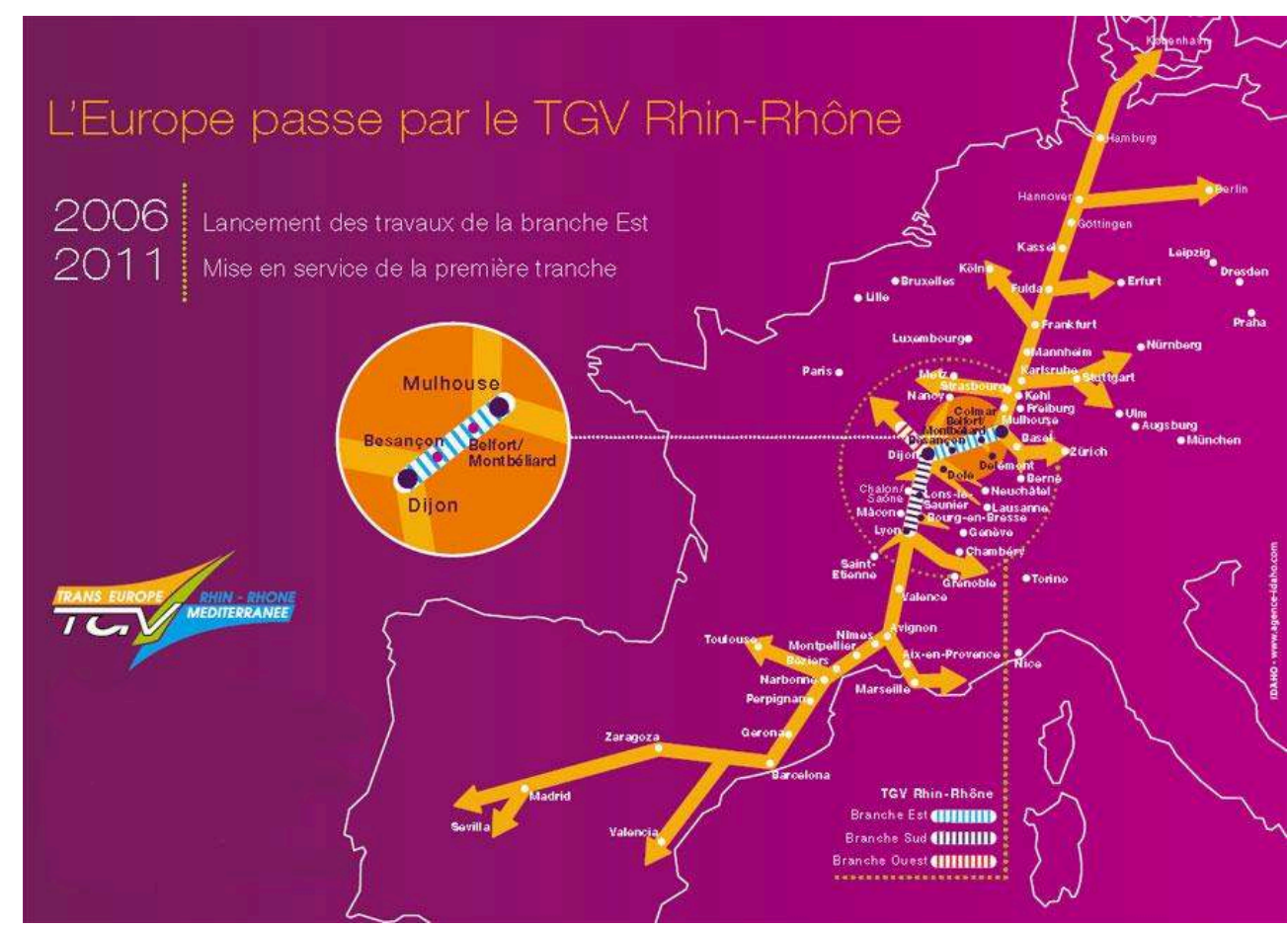

11 Si l'on compare la vision marketing du projet à la réalité des flux, la différence saute aux yeux (Figure 1). Les flux sont principalement jacobins avec des trains qui se remplissent en direction de Paris et qui se vident lorsqu'on s'en éloigne. Au quotidien, il n'y a que $6 \mathrm{TGV}$ pour Lyon et 3 pour Marseille contre 16 pour Paris (17 en comptant le TGV de Roissy CDG). L'effet centralisateur est encore renforcé par les Lyria qui traversent le Jura. Les réalités du marché français fondées sur le tropisme parisien restent donc primordiales.

12 A l'évidence, l'objectif initial pourra être atteint le jour où fonctionnera la branche Sud vers Lyon. Or cette branche n'a ni financement, ni tracé, ni calendrier.

\section{Le TGV Rhin-Rhône est-il nécessaire?}

La question peut paraître iconoclaste mais la SNCF elle-même la pose de manière indirecte sur son site internet de vente. Taper Mulhouse-Paris donne comme résultat un grand nombre de connexions, soit par le TGV-Rhin-Rhône, soit par le TER vers Strasbourg puis le TGV-Est en correspondance. Bien entendu, le TGV drapeau, celui du meilleur temps de parcours, fait pencher la balance en faveur du Rhin-Rhône avec $2 \mathrm{~h} 40$ contre 3 h19 sur Mulhouse-Paris (Figure 3). Mais seuls les six TGV Lyria venant de Suisse se trouvent dans ce cas de figure. Outre Mulhouse, les neuf autres TGV s'arrêtent à Belfort/Montbéliard et/ou Besançon, parfois à Montbard (Côte-d'Or). Le temps de parcours le plus courant est donc de 2 h55 sur Mulhouse-Paris. Avec seulement 11 minutes de moins, il est presque équivalent aux 3 h06 du TGV-Est drapeau qui circulait de juin 2007 à décembre 2011 entre Mulhouse et Paris via Strasbourg. Depuis l'introduction du TGV Rhin-Rhône, il reste possible de prendre un TER entre Mulhouse et Strasbourg, où, grâce à une correspondance rapide, on arrive finalement à Paris en 3h19 au total. On peut dire affirmer que Strasbourg, Mulhouse et Colmar bénéficient 
désormais d'une excellente connectivité, c'est-à-dire du choix entre deux liaisons et deux gares parisiennes avec des temps de parcours assez proches.

Mais la messe des temps de parcours n'est pas encore entièrement dite. Les travaux d'achèvement de la LGV-Est entre Baudredourt (Moselle) et Vendenheim (Bas-Rhin) ont été engagés en 2011, notamment avec le percement du tunnel de Saverne sur $4 \mathrm{~km}$ de long. Fin 2015, ou peut-être un peu plus tard, on gagnera une demi-heure entre Paris et Strasbourg, avec un temps de parcours de 1h50. A cette date, Mulhouse-Paris par le Rhin-Rhône sera toujours à $2 \mathrm{~h} 40$. Un Mulhouse - Paris via Strasbourg se fera en $2 \mathrm{~h} 49$, correspondance incluse. Si l'on remettait un TGV-Est à Mulhouse, on tomberait à $2 \mathrm{~h} 36$ !

Figure 3 : La liaison Paris-Belfort, meilleurs temps

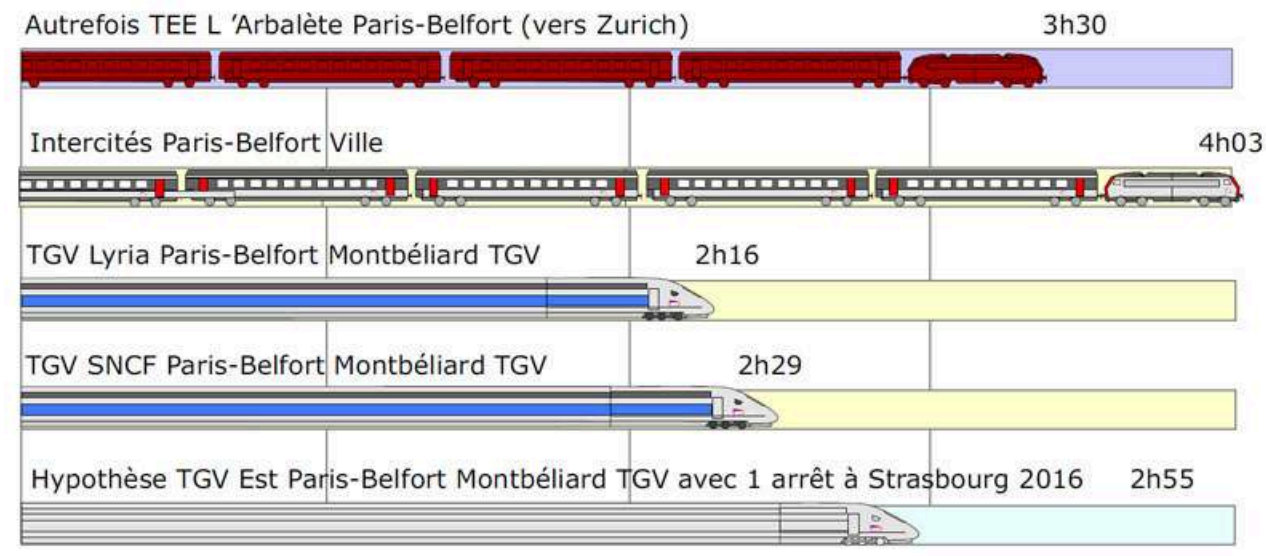

Données : SNCF. Service d'été 2014 Calcul personnel pour 2016

R. Woessner ENeC Paris Sorbonne

Les autres villes desservies ne gagnent pas de temps avec le TGV Rhin-Rhône à l'exclusion notable de Belfort et de Montbéliard où l'amélioration est très significative. Il faut 4 h08 au vieux Belfort-Paris dans le meilleur des cas contre $2 \mathrm{~h} 16$ au TGV. Ce qui revient à dire que la LGV Rhin-Rhône n'aura de sens qu'avec l'achèvement des travaux selon la vision développée en son temps par l'association TGV Rhin-Rhône Méditerranée.

IV. Quels sont les périls pour l'achèvement de la LGV Rhin-Rhône?

17 Il reste de l'ouvrage, avec d'une part les parties Est et Ouest de la branche Est autorisées par la Déclaration d'utilité publique de 2002, d'autre part la branche Ouest et la branche Sud.

On peut jouer sur les mots: vu de Strasbourg, le tronçon Est de la branche Est Lutterbach (Haut-Rhin) - Petit-Croix (Territoire de Belfort) de $35 \mathrm{~km}$ apparait volontiers comme un élément de la branche Sud puisqu'il permettrait de gagner du temps entre Strasbourg et Lyon (Figure 4). En évitant Mulhouse, ce tronçon apporterait un gain de temps de 26 minutes sur Strasbourg-Lyon. On comprend dès lors pourquoi la Région Alsace, la Ville et la Communauté Urbaine de Strasbourg font le forcing pour ce grand travaux que $\mathrm{M}$. Richert, président de la région Alsace et ministre des collectivités du gouvernement Fillon, a promis de faire engager en 2014 et peut-être même en 2013. En attendant la concrétisation de cette promesse, et malgré l'opposition de la Chambre 
de Commerce et d'Industrie (CCI) de Mulhouse-Sud-Alsace, on a construit en 2011 le shunt (pour Réseau Ferré de France (RFF), le «raccordement court») qui, via le Cokrouri et le franchissement du canal du Rhône-au-Rhin, permet d'éviter la gare de Mulhouse et son arrêt (Figure 8). La position de la CCI mérite une explication : elle est favorable à l'achèvement de la LGV entre Lutterbach et Petit-Croix; elle admet la possibilité que des trains passent au nord-ouest de Mulhouse; dès lors, dit-elle, pourquoi avoir dépensé plus de 50 millions d'euros pour un ouvrage qui deviendrait rapidement inutile?

En 2013, la convention de financement devrait être signée pour terminer la branche Est. Les $35 \mathrm{~km}$ de la partie alsacienne coûteraient 850 millions d'euros et les $15 \mathrm{~km}$ de la Côte-d-Or 310 millions, soit respectivement 24,3 et 20,6 millions au km. Au niveau des collectivités, l'Alsace verserait $26,5 \%$ du total, la Franche-Comté $13,5 \%$ et la Bourgogne $10 \%$, soit $50 \%$ du total. Rhône-Alpes a signifié son refus de participer au financement. On ne sait pas encore quelle pourrait être le montant des contributions de l'UE, de l'État français, de la Suisse et de l'Allemagne. Pour la première phase de la branche Est, RFF avait apporté $28 \%$ de l'investissement mais affirme désormais vouloir se limiter à $10 \%$.

Figure 4 : La liaison Strasbourg-Lyon, meilleurs temps

TGV Rhin-Rhône Strasbourg-Lyon meilleur temps (arrêts à Mulhouse, Dijon, Chalon) 3h40

\begin{tabular}{|l|l|}
\hline \hline TGV Rhin-Rhône Strasbourg-Lyon sans arrêt décembre 2012 \\
\hline \hline
\end{tabular}

Données : SNCF. Association TGV Rhin-Rhône Méditerranée R. Woessner ENeC Paris Sorbonne

En Côte-d'Or, la réalisation de la partie ouest de la branche Est (15 km entre Genlis et Villers-les-Pots) et de la branche Ouest (Dijon - Aisy) apparait problématique (Figure 5). Il n'existe ni calendrier ni financement pour les travaux. Toutefois, on peut compter sur un lobbying actif de la part des Dijonnais en vue de leur réalisation. Pour JeanJacques Bavoux (1993), «les efforts inlassables pour détourner et capturer infrastructures et circulations constituent indéniablement l'un des ressorts fondamentaux de l'histoire dijonnaise ». Or Dijon risque de se faire éviter par la branche Sud. Elle l'est déjà en partie par la virgule de Perrigny qui contourne la ville par le sud-est. Et au quotidien trois TGV Lyria zurichois sur six ne s'arrêtent pas dans sa gare bien qu'ils soient obligés de la traverser à petite vitesse ; on atteint là le comble de l'obsession de la grande vitesse à tout prix ! 
Figure 5 : Dijon et les LGV

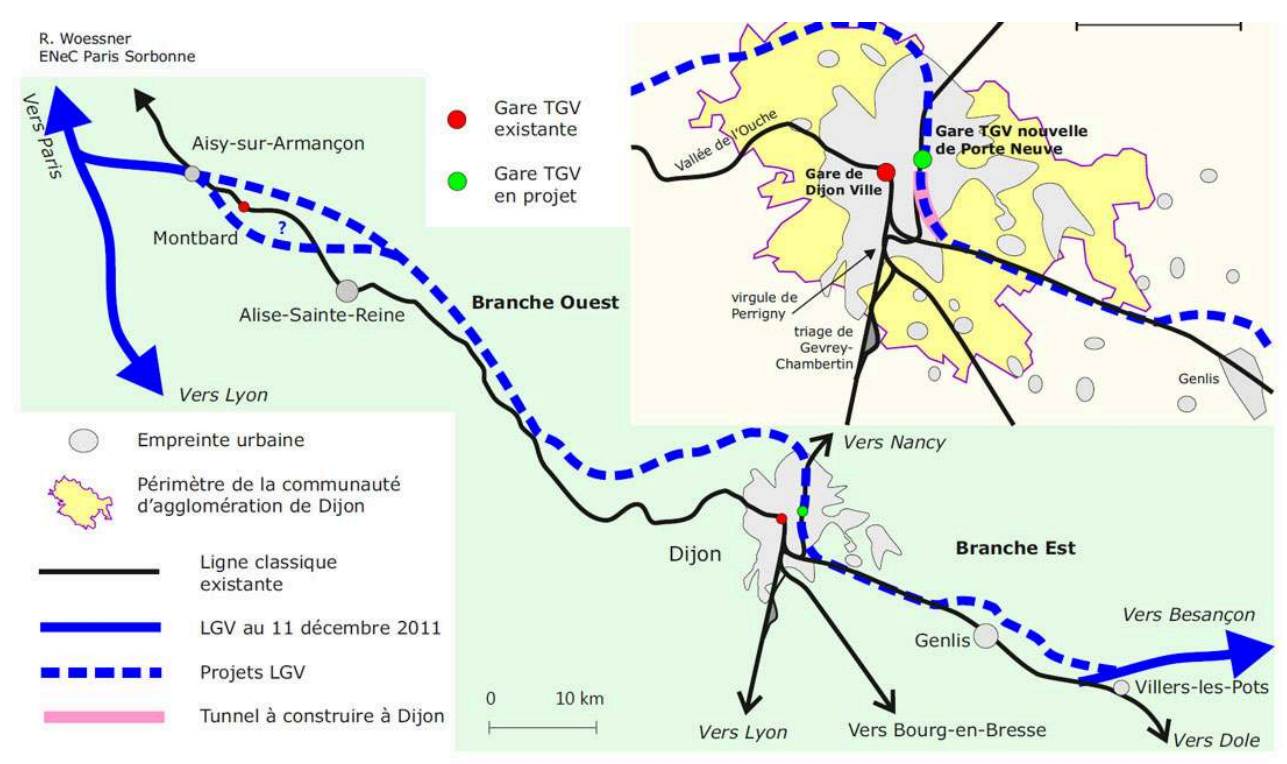

Il reste le problème de la branche Sud qui a le soutien de l'Union européenne et du Grenelle Environnement mais qui ne dispose ni d'un tracé définitif, ni d'un financement, ni d'un calendrier pour sa réalisation. De nombreuses variantes ont déjà été proposées pour son tracé. Elle est en outre empêtrée dans un débat sur sa fonctionnalité : doit-elle être uniquement une LGV ou également une ligne fret ? Depuis 2008, les études les plus récentes privilégient l'idée d'une ligne mixte, à l'instar des réflexions en cours entre Lyon et Turin, ou encore entre Nîmes et Montpellier. Dans ce cas, les TGV ne dépasseraient pas la vitesse de $270 \mathrm{~km} / \mathrm{h}$. Beaucoup de questions se posent quant à cette cohabitation des trains de fret et de la grande vitesse. Les premiers sont parfois accusés de freiner les seconds. En outre, malgré des tentatives de relance depuis le début des années 2000, le fret ferroviaire français ne cesse de perdre des marchés. Quant à l'attitude des élus, ce n'est pas l'enthousiasme en Bourgogne qui craint de se faire éviter au profit de Dole. Les Bourguignons préfèreraient une modernisation de la ligne historique Dijon - Chalon-sur-Saône - Lyon avec de meilleurs temps de parcours.

Le Grenelle Environnement a fait apparaitre un nouveau péril pour la LGV Rhin-Rhône. Il a remis en selle le projet Centre-France qui n'était plus évoqué depuis une vingtaine d'années. Cette liaison à grande vitesse irait de Paris-Austerlitz à Lyon via ClermontFerrand. La SNCF et RFF la soutiennent. En effet, à Paris, la gare d'Austerlitz, qui conduit vers le Massif Central, dispose de réserves de capacités, alors que la gare de Lyon devrait être "saturée à court et à moyen terme" selon l'Atelier Parisien d'Urbanisme (APUR, 2009). Le projet Centre-France peut compter sur des élus qui peuvent peser de tout leur poids tandis que la branche Sud du TGV Rhin-Rhône a besoin de soutiens en Allemagne, en Suisse et en Espagne, ce que l'État français risque de moins entendre. Or seule la branche Sud donnerait sa légitimité interrégionale et européenne au TGV Rhin-Rhône.

Depuis 2009, RFF soutient prioritairement un tracé pour la branche Sud qui serait parallèle à l'autoroute A39 sur quasiment tout son parcours, donc au plus près de la ligne historique du Revermont (Figure 6). La LGV éviterait ainsi les zones naturelles fragiles du Val de Saône, de la Bresse et des Dombes, où sont établis de belles forêts et 
de nombreux étangs propices à la biodiversité et aux oiseaux migrateurs. Cela étant, certains spécialistes estiment que l'association autoroute / LGV crée une barrière trop difficile à franchir pour la faune terrestre, la confinant ainsi dans des territoires restreints et nuisant à leur mode de vie comme à leur reproduction. En outre, suivre le tracé de l'autoroute impliquerait des contraintes techniques; les pentes relativement fortes nuisent au fret et les courbes un peu serrées ne sont pas propices à la très grande vitesse.

Figure 6 : La branche Sud et le bouchon lyonnais

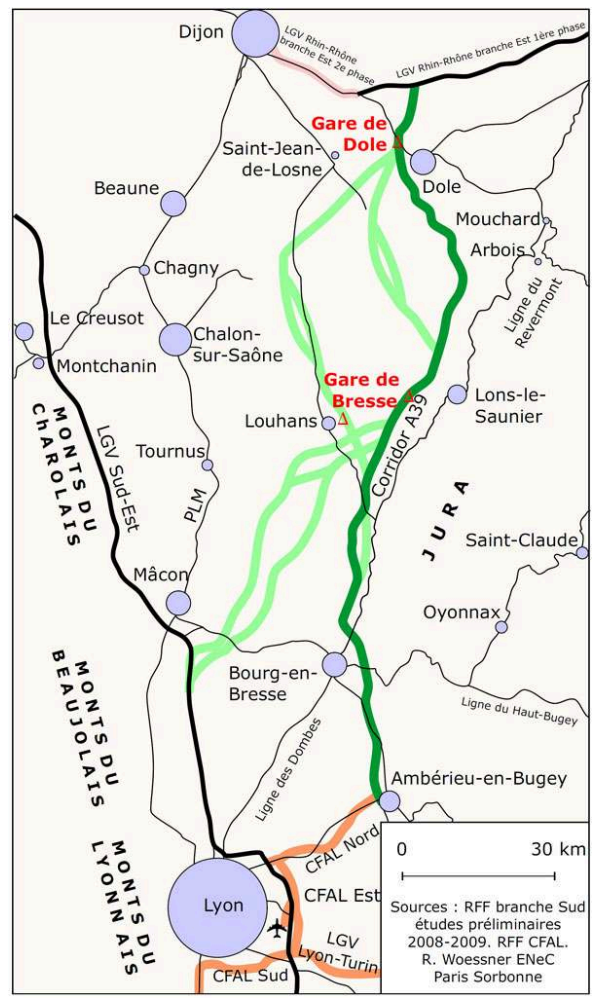

Enfin, il faut s'intéresser au Contournement Ferroviaire de l'Agglomération de Lyon (CFAL), un projet de $70 \mathrm{~km}$ de long qui, depuis 2011, est arrivé au stade des études d'avant-projet. Il vise à désengorger l'agglomération lyonnaise. Il est vital pour le TGV Rhin-Rhône à qui il ouvrirait les portes de la Méditerranée et des Alpes. Le CFAL se connecte aussi sur le projet de LGV Lyon-Turin. Au total, en mettant tous les maillons bout à bout entre Alsace / Lorraine et Rhône-Alpes / PACA, la France pourrait créer une alternative à la traversée des Alpes par la Suisse, aussi bien pour le fret que pour la grande vitesse voyageurs. Du point de vue des infrastructures, le Grand Est français serait alors à niveau pour affronter la concurrence européenne.

Dans le même ordre d'idées, d'autres maillons manquants et éloignés apparaissent stratégiques pour le TGV Rhin-Rhône. Vers le sud, c'est le cas de Nîmes-Perpignan et de la traversée souterraine de Barcelone; d'Aix-en-Provence - Nice. Vers le nord, l'Allemagne a commencé à construire une LGV entre Stuttgart et la Bavière ; et le projet de contournement contesté de Mannheim via un tunnel permettrait de rejoindre facilement Francfort-sur-le-Main qui se trouve aujourd'hui à deux heures de train de Strasbourg. 
Une dernière réflexion, mais non des moindres, concerne une éventuelle liaison vers la Lorraine via la Haute-Saône (Mignot, 2011). Aujourd'hui il faut compter $2 \mathrm{~h} 20$ pour parcourir $210 \mathrm{~km}$ entre Nancy et Dijon. A partir de la LGV existante, les TGV pourraient alors éviter le corridor lotharingien, profiter de la LGV Rhin-Rhône avec une branche nouvelle vers Lure, et de là retrouver Nancy avec une liaison modernisée via Epinal.

\section{Avec un projet de deux gares, les Dijonnais sont-ils dans l'erreur?}

Sur la desserte Rhin-Rhône inaugurée en 2011 se trouvent trois gares historiques Dijon, Besançon-Viotte et Mulhouse - et deux gares nouvelles - Besançon FrancheComté TGV à Auxon (Doubs) et Belfort-Montbéliard TGV à Meroux (Territoire de Belfort).

28 A Dijon et à Mulhouse la situation est relativement simple à ce jour mais l'avenir de leurs gares est loin d'être tracé. On a là deux ensembles urbains de taille comparable : 336.000 habitants pour l'aire urbaine de Dijon et 281.000 pour celle de Mulhouse (2008). En tant que capitale régionale proche de Paris, Dijon a sans doute un potentiel de clientèle plus important que Mulhouse. Mais les deux villes ne sont pas des métropoles, Dijon est au 24e rang des aires urbaines françaises et Mulhouse au 34e rang. Il leur faut donc se battre pour pouvoir exister. Il faut également que leurs gares soient alimentées par des TER qui permettent de rabattre de la clientèle vers les TGV. Dijon reçoit 83 TER par jour ouvrable et Mulhouse 153 en comptant les trams-trains (service d'hiver 2011-2012 3 ) et en attendant l'arrivée de nouvelles liaisons depuis Fribourg-en-Brisgau. Mulhouse est donc bien mieux lotie que Dijon : elle reçoit pratiquement deux fois plus de TER que Dijon, en général sur des distances-temps plus courtes, dans un environnement régional et transfrontalier de villes très peuplées. Mais au final Dijon reste tout de même le nœud principal du système Rhin-Rhône avec 26 TGV quotidiens qui s'y arrêtent, contre 20 pour Mulhouse. Il est vrai que la capitale bourguignonne récupère les flux lorrains et vaudois qui lui apportent 9 arrêts de TGV par jour (Figure 1).

Lors de l'inauguration de la LGV en 2011, les discours des élus ont été lucides. Personne n'attend plus de «miracle TGV » qui produirait des effets automatiques de croissance économique et démographique auxquels les chercheurs ont renoncé depuis longtemps (Offner, 1993). Tout le monde est convaincu que le TGV ne constitue qu'une opportunité qu'il s'agit de valoriser. Il faut donc exploiter l'amélioration de l'accessibilité par la construction de pôles de croissance situés à proximité des gares. Des entreprises comme des services publics de toutes sortes pourraient alors s'y agglomérer.

30 A Dijon, le quartier de la gare historique n'offre guère d'opportunités foncières (Figure 5). A long terme, les TGV devraient utiliser la gare de Porte-Neuve, où passent aujourd'hui de rares TER et trains de fret. On se priverait donc d'une correspondance commode entre les TGV et les TER. Les deux gares se situent à équidistance du Palais des Ducs. En son temps, la construction de la gare historique de Dijon Ville avait bouleversé le tissu urbain et l'économie de la ville; les fonctions supérieures du XIXe siècle s'étaient implantées à proximité de la place Darcy en développant des axes urbains en continuité avec la gare. Aujourd'hui, le quartier de Porte-Neuve délaissé par 
les militaires et les transporteurs de marchandises offre les disponibilités foncières requises. Un nouveau quartier d'affaires et de culture est déjà sorti de terre: auditorium, CCI, hôtels, bureaux, tour à énergie positive... Mais le fait de tabler sur deux gares cassera l'effet d'alimentation du TGV par les TER que le tramway (couleur cassis...), qui les reliera entre elles, est censé préserver depuis l'automne 2012.

\section{La gare de Mulhouse, un pôle de croissance?}

A Mulhouse, les travaux ont commencé à modifier les alentours de la gare centrale. En 2009, M. David Mangin est devenu l'urbaniste-conseil chargé de développer la ZAC de la gare. Spécialiste des espaces publics, il oppose «la ville passante» aux logiques d'enfermement et de non-mixité sociale. Il a commencé à traiter un périmètre de 23 ha, emprise SNCF comprise, fort de 4 hectares de surfaces constructibles. La ZAC s'étire le long des voies ferrées et du canal Rhin-Rhône à petit gabarit; le vis-à-vis de la gare a reçu la sous-préfecture en 2011 dans les anciens locaux de la Société Commerciale des Potasses d'Alsace. Un parking de 450 places (on paye 10 euros pour 24 heures) et un immeuble de trois étages ont été mis en service. Les bureaux du diéséliste finlandais Wärtsilä se sont installés dans ce bâtiment, après avoir quitté le site historique de la SACM. Les constructions suivantes viendront au fur et à mesure des besoins. Ainsi la prudence s'impose. Selon un expert immobilier, il y a $66.800 \mathrm{~m}^{2}$ de bureaux immédiatement disponibles dans la ville, soit environ $10 \%$ du total existant ${ }^{4}$.

Figure $7:$ Mulhouse et ses gares

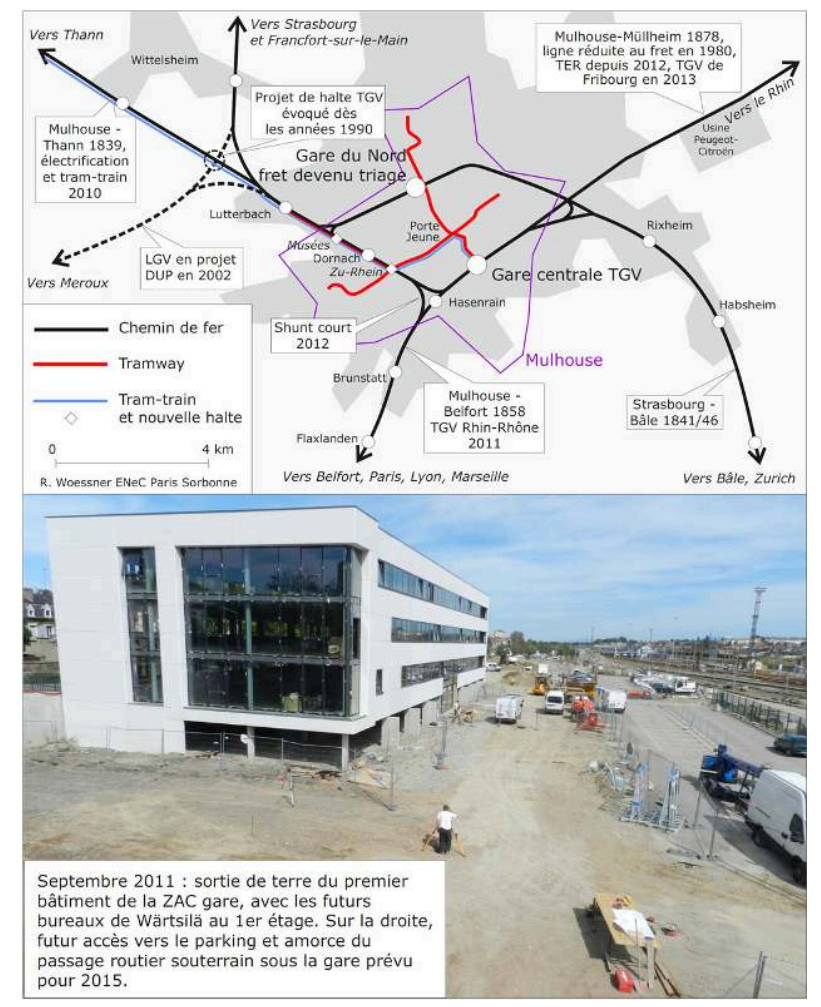

32 A long terme, Mulhouse Alsace Agglomération (m2A) souhaite créer une halte TGV à Lutterbach dans l'espoir de capter des arrêts pour les trains qui éviteront l'agglomération (Figure 7). En se limitant à un simple arrêt, les ambitions sont 
modestes. La m2A veut éviter un effet de vampirisation de la gare centrale. Placé en lisière de la ville et de la forêt du Nonnenbruch, disposant de réserves foncières, le site lutterbachois est traversé par la ligne TER et tram-train de Thann; la RN66 à 2X2 voies y conduit naturellement. Toutefois, le projet de prison départementale n'y est sans doute pas la meilleure vitrine que l'on puisse espérer.

\section{Le syndrome des betteraves menace-t-il les gares nouvelles?}

En Franche-Comté, l'aire urbaine de Besançon compte 243.000 habitants $(2008$ - 41e rang français), celle de Montbéliard 180.000 (45e rang) et celle de Belfort 105.000 (71e rang). En termes de pouvoir d'achat, Besançon, capitale régionale, est bien plus favorisée que les villes industrielles du Nord-Est Franche-Comté.

L'accessibilité des gares TGV franc-comtoises est pour le moins compliqué. A Besançon, pour RFF comme pour la SNCF, il n'a pas été question de faire passer la LGV par la gare centrale de Viotte, une desserte qu'elles ont jugée chronophage ; le karst de la forêt de Chailluz avait également été évoqué en tant que difficulté technique. C'est pourquoi 15 TER relient la nouvelle gare TGV d'Auxon à la gare centrale de Viotte en 12 minutes grâce à un tronçon à voie unique mais entièrement rénové de l'ancienne ligne VesoulBesançon.

A Belfort-Montbéliard TGV, il n'existe pas encore de desserte TER. Il faudra rouvrir la ligne à voie unique Belfort-Delle fermée aux voyageurs depuis 1992 (Figure 8). Une étude de 2006 préconise une liaison allant de Bienne, de l'autre côté du Jura en Suisse, jusqu'à Lure (Haute-Saône) et même jusqu'à Epinal (Vosges) faisant ainsi la jonction avec le TGV-Est. Longtemps, on espérait un TER à Meroux pour l'inauguration de la LGV Rhin-Rhône. Mais le devis prévu par le Contrat de Plan Etat-Région 2000-2006 prévoyait un coût de 64 millions d'euros, qui est passé à 102 millions en 2010, notamment parce que 20 passages à niveau doivent être supprimés. La Suisse a budgété 20 millions de FS. 
Figure 8 : La gare de Meroux

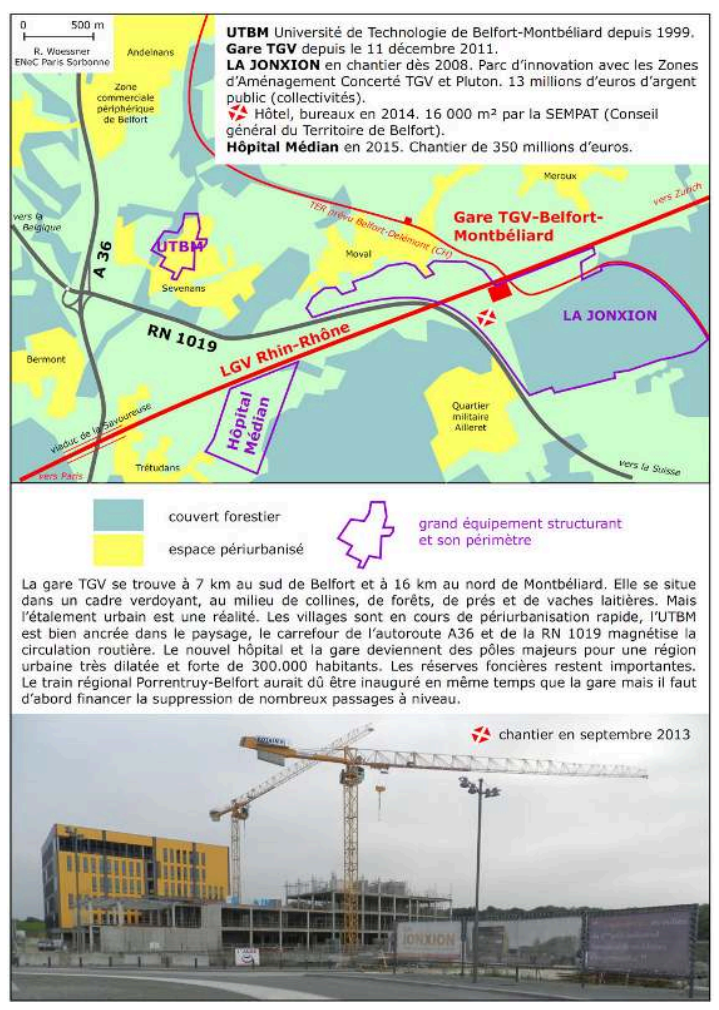

En comparaison des gares nouvelles de la ligne du TGV-Est, celles de Besançon et de Belfort-Montbéliard apparaissent comme étant de belles dimensions. Pour la gare implantée dans la forêt d'Auxon, les aspects patrimoniaux ont la partie belle : en créant une muraille percée de petites fenêtres et des glacis enherbés, l'architecte Jean-Marie Duthilleul rend un hommage à Vauban; on sait que la ville est inscrite avec d'autres au Patrimoine mondial de l'UNESCO grâce à cette architecture militaire. Une énorme horloge mise au point par l'université décore le hall. Chauffée au bois, la gare est labellisée " haute qualité environnementale et basse consommation d'énergie », ce qui est inédit pour une gare TGV. Besançon affiche de grandes ambitions pour la gare d'Auxon: "D'ici 20 ans, [..] 3.500 emplois seront créés sur une surface totale d'environ $92.000 \mathrm{~m}^{2}$ de locaux et bureaux principalement dans le secteur high-tech et la recherche. Le site, dans un écrin de verdure et respectant une distance d'au moins de $200 \mathrm{~m}$ avec les premières habitations, prendra forme progressivement sur 25 ha entre la gare et l'actuelle zone artisanale d'Auxon-Dessus $»^{5}$.

A Meroux, la gare attend 1,1 million de passagers annuels; elle dispose de 1.100 places de parking (payantes) ${ }^{6}$. Accolée à la gare, la ZAC Jonxion prévoit 150 ha de terrains pour des activités de type tertiaire ou pour de petites industries innovantes "pouvant accueillir 2.000 salariés $»^{7}$. Un peu par le fait du hasard des opportunités et des décisions politiques, le nouvel hôpital de l'Aire urbaine sera construit à proximité. La gare de Meroux est également proche du site universitaire de Sevenans (UTBM) ainsi que du carrefour de l'autoroute A36 et de la N19.

De fait, les promoteurs des gares nouvelles franc-comtoises sont fermement décidés à éviter le syndrome des betteraves tel qu'on l'observe en Picardie à AblaincourtPressoir, où une petite gare et son parking sont isolés au milieu des champs sans que l'on puisse déceler d'effet d'entraînement depuis son ouverture en 1994. Si elles 
réussissent, les gares de Besançon et de Belfort-Montbéliard vont renforcer le mouvement de périurbanisation, d'étalement urbain et de consommation voire de mitage de l'espace. Le risque est alors d'urbaniser mais sans urbanité, c'est-à-dire sans créer de lieux où les gens vivent, se rencontrent et accèdent facilement à divers services. Un piège se referme-t-il sur les villes les plus importantes de la FrancheComté? Les urbanistes de l'Aire urbaine en sont conscients. Dans leurs schémas prospectifs, ils imaginent volontiers un anneau ferroviaire reliant Belfort, Héricourt, Montbéliard, la gare TGV et à nouveau Belfort, un anneau qui structurerait la Porte de Bourgogne et favoriserait les mobilités fondées sur les transports en commun (Woessner, 2008). Ce qui, au pays de Peugeot, est une vraie gageure. En attendant cet avenir idéalisé et lointain, Montbéliard comme Belfort proposent deux navettes d'autocar avec de bonnes fréquences vers la gare TGV au prix du ticket urbain $(1,50$ euro).

\section{La Métropole Rhin-Rhône, un concept mort-né ?}

Depuis des décennies, il est question de créer des réseaux urbains en France, aussi bien pour rééquilibrer l'armature urbaine du pays que pour y développer les fonctions supérieures. On se souvient du fiasco des métropoles d'équilibre, une opportunité historique créée par la DATAR en 1965, court-circuitée par le référendum négatif de 1969 et finalement rejetée par le président Pompidou. En 1994, la DATAR recommandait de créer des réseaux urbains (DATAR, 1994). Une tentative locale avait émergé avec le Réseau de Villes Rhin-Sud dès 1992 avec Belfort, Colmar, Héricourt, Montbéliard, Mulhouse et Saint-Louis. Ce réseau avait permis la création du Pôle Véhicule du Futur, mais il a néanmoins disparu faute d'autres réussites. En 2007, dans le cadre national des contrats métropolitains, est venue la Métropole Rhin-Rhône (MRR) avec Bâle, Belfort, Besançon, Chalon-sur-Saône, Dijon, Le Creusot-Montceau, Montbéliard, Mulhouse et Neuchâtel. Les travaux engagés de la LGV avaient alors conduit à une évidence : il faut fédérer les villes pour créer un effet-TGV.

«Si [la Franche-Comté] n'est pas pauvre, elle n'est pas riche pour autant » écrivent les géographes Pascal Bérion et Marina Duféal (2006). C'est bien là le problème de l'espace Saône-Rhône qui a pu se développer tant que l'industrie prospérait. Mais aujourd'hui, de manière générale, un nombre restreint de métropoles tend à se substituer aux autres villes en tant que places centrales. Avec la mondialisation, les emplois supérieurs prennent davantage d'importance, et cela au profit des métropoles où ils s'agglomèrent de préférence. En 1999 déjà, l'agglomération de Lyon rassemblait environ 76.000 emplois métropolitains supérieurs, à comparer avec les 49.000 contenus dans l'ensemble de la Bourgogne, de la Franche-Comté, de l'Ain et du Sud-Alsace.

41 A l'échelle de l'histoire, les villes de Rhin-Rhône ne fonctionnent pas en réseau. Elles se sont même fréquemment opposées entre elles. En effet, dans le contexte de la logique jacobine, elles ont cherché à s'attirer les bonnes grâces du pouvoir central autant pour espérer un développement économique que pour fixer des activités administratives d'importance. A ce jeu, un voisin était aussi un concurrent et les relations de bon voisinage - on n'ose évoquer l'idée de coopération - n'avaient guère d'intérêt (Woessner, 2008). Aujourd'hui, les divisions administratives et politiques génèrent toujours de nombreuses coupures, alors que les périmètres de projet débordent au-delà des limites des administrations. La situation devient vite compliquée lorsque chacun 
voit midi à sa porte. Ainsi, le Contrat compensatoire à l'abandon du grand canal RhinRhône (1999-2006) n'avait vu émerger aucun projet interrégional. Le "Rhin-Rhône d'acier » (le fret ferroviaire) appelé de leurs vœux par les écologistes comme par les CCI n'a pas non plus été réalisé. Le tourisme fluvial n'a pas été développé, à l'exception d'aménagements en pointillé, comme certains segments de la "véloroute NantesBudapest ", mais avec quels effets?

Enfin, les frontières nationales, certes de plus en plus poreuses, perdurent néanmoins lorsqu'il s'agit de systèmes d'organisation. La dimension transfrontalière apporte un niveau supplémentaire avec d'autres règles, comportements et représentations qu'il faut comprendre, assimiler et gérer. Du côté français, le préfet joue un rôle éminent et en réfère à Paris ; du côté allemand ou suisse, le fédéralisme accorde une grande place à l'échelon local qui entretient des relations de proximité avec le pouvoir. En Suisse en particulier, les cantons et les communes ont les coudées franches pour de nombreuses réalisations, à l'intérieur comme à l'extérieur des frontières.

Sans surprise, les villes de la LGV Rhin-Rhône entretiennent peu de relations. Presque tout serait à faire pour les susciter ou les développer. Selon les enquêtes mobilité de $2009^{8}$, « la mobilité interurbaine ne dessine pas un territoire partagé où chaque pôle du réseau métropolitain Rhin-Rhône aurait des relations privilégiées avec ses semblables. Les échanges se font avec les pôles voisins et avec Paris. [..] De plus, étant donné l'éloignement des gares TGV de Besançon et de Belfort-Montbéliard et les coûts largement supérieurs des abonnements TGV par rapport à ceux des trains régionaux (TER), les navetteurs de l'axe Rhin-Rhône ne devraient pas changer leurs habitudes. Parmi les relations entre pôles du réseau métropolitain, la liaison Dijon-Mulhouse est l'une des seules susceptibles de croître sensiblement après la mise en service du TGV Rhin-Rhône » (Richer, Bérion, 2010).

De fait, dans le temps long, trois scénarios semblent crédibles sur l'axe Saône-Rhin (Figure 9).

Figure 9 : Itération vers la systémogenèse

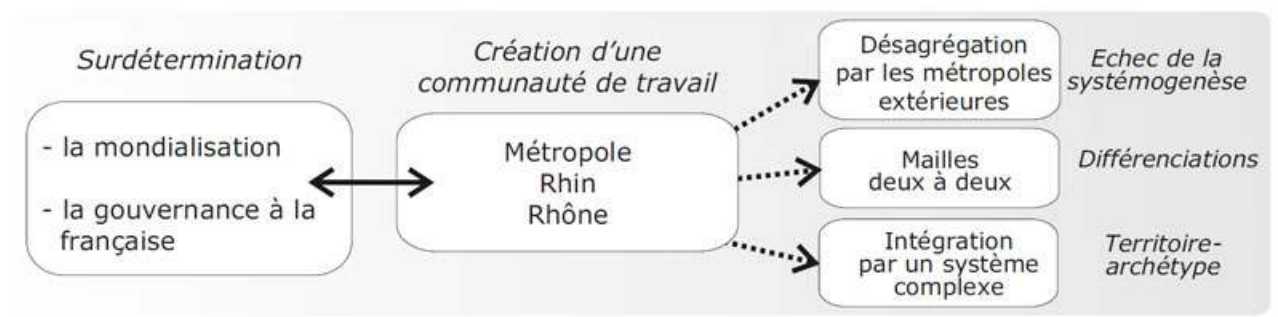

Le scénario de la désagrégation voit les métropoles extérieures à la MRR augmenter leur influence. Dans ce cas de figure, Paris, Lyon, Strasbourg, Bâle et Fribourg-enBrisgau rassemblent les forces vives de la croissance et de l'innovation. Elles désarticulent les modestes réseaux régionaux présents sur leurs marges. Ainsi, Strasbourg jouerait la carte de l'Europe institutionnelle; les avions et les TGV la relieraient aussi vite que possible aux eurocités. Le Sud-Alsace deviendrait une banlieue de Bâle où la partie française de l'Eurodistrict Trinational de Bâle (ETB) profiterait de la localisation de fonctions supérieures. La zone d'influence de Lyon absorberait le sud de la Saône-et-Loire ainsi que la partie méridionale du Jura. Les Régions Alsace, Bourgogne et Franche-Comté n'auraient plus de cohérence interne. Aucun système d'ensemble ne serait plus envisageable. Les effets de type fractal se multiplieraient: à leur échelle, 
toutes les villes essaieraient de consolider leur zone d'influence, systématiquement grignotée et affaiblie par les villes voisines plus importantes. Le mode de gouvernance fondé sur les EPCI et les Pays tournerait à l'aigre. Lent mais irréversible, le déclin économique et démographique s'étendrait à partir des cœurs industriels et des poches enclavées de la ruralité.

Le scénario des mailles deux à deux fait apparaître la MRR comme une chaîne donnant l'illusion de la continuité réticulaire. Les villes s'organiseraient alors entre voisines immédiates pour créer des duopôles, des couples urbains formant une succession de maillons. Les EPCI, les Pays et les eurodistricts créeraient des contrepoids face à la concurrence des métropoles extérieures. Dijon et Besançon formeraient une sorte de supercapitale dédoublée pour la Bourgogne et la Franche-Comté; cette tendance est déjà entamée pour certaines administrations déconcentrées ainsi qu'avec la création du Pôle de Recherche et d'Enseignement Supérieur (PRES) commun aux deux universités. Plus au nord, un deuxième duopôle est possible notamment parce que l'entreprise Peugeot-Citroën a créé de forts effets structurants entre Belfort-Montbéliard et Mulhouse ; mais une communauté de problèmes risque d'apparaître si le Pôle Véhicule du Futur ne parvient pas à sortir les entreprises de la culture de la seule production industrielle. Un troisième maillage concernerait le Jura avec une croissance posthorlogère et transfrontalière. Les villes du piémont suisse sont riches de fonctions de commandement et d'innovation; la partie française du Jura pourrait devenir autre chose qu'un réservoir d'activités et de salariés à moindre coût.

Enfin, le scénario le plus volontariste, celui de l'intégration généralisée, absorbe et dépasse le précédent. L'espace Rhin-Rhône ne se décomposerait plus en sous-systèmes mais il construirait un système complexe où tout le monde collaborerait avec tout le monde. Ceci signifie qu'une décision ou qu'un événement local aurait des conséquences dans l'ensemble du réseau. Ainsi, en réunion publique, le vice-président de la Communauté d'agglomération de Dijon a déjà évoqué «Dijon, ville rhénane " ${ }^{9}$ La MRR n'aurait rien d'insulaire. Elle développerait également des portes d'entrée multiples et des liaisons aussi efficaces que plurielles. Le transit introduirait des problématiques communes : comment éviter l'effet-tunnel et retenir les flux ? Les perspectives reposeraient sur le maillage des autoroutes et des voies express (l'autoroute transjurane est toujours en chantier), sur la grande vitesse ferroviaire française, allemande et suisse, sur le projet de canal à grand gabarit qui redresse la tête, sur une desserte fine et intermodale du territoire. L'EuroAirport, à qui il manque un accès ferroviaire, deviendrait la porte ouverte sur la mondialisation (tout comme le feraient les ports rhénans et saônois pour les marchandises). La région deviendrait créative : les industries agro-alimentaires, le monde de l'automobile, les nano- et les biotechnologies rebondiraient sous des formes d'endogénéisation territoriale fondées sur des clusters; les nouvelles technologies irrigueraient l'ensemble de ces secteurs d'activité. Aucune ville n'aurait la primauté au sein d'un réseau urbain multipolaire. Toutefois, l'agglomération bâloise se placerait un pas devant les autres parce qu'elle est déjà une ville-monde (avec ses banques et assurances, la logistique, le tourisme fluvial, l'art contemporain, les architectes, les foires et les salons, les biotechnologies et la bigpharma).

48 Mais l'application locale de la loi du 16 décembre 2010 portant sur la réforme des collectivités territoriales a ruiné ce troisième scénario. Entre autres dispositions, elle crée un nouvel échelon territorial, le "pôle métropolitain ». Celui-ci «regroupe des 
établissements publics de coopération intercommunale à fiscalité propre formant un ensemble de plus de 300.000 habitants. L'un d'entre eux compte plus de 150.000 habitants " (Article L5731). Le pôle métropolitian peut intégrer des compétences jusqu'ici dédiées aux départements, aux régions et même à l'Etat. Par arrêté préfectoral du 21 décembre 2011, Strasbourg et Mulhouse créent le premier pôle métropolitain français. Mulhouse fait donc une volte-face spectaculaire en se détournant de la MRR qu'elle avait pourtant appelée de tous ses vœux. Désormais, que vont faire les villes comtoises et bourguignonnes? Et comment la Suisse perçoit-elle cette nouvelle configuration?

De fait, le pôle métropolitain Strasbourg-Mulhouse corrobore le premier scénario, celui de la métropolisation. Strasbourg - c'est-à-dire la Ville, la Communauté urbaine et la Région - s'intéressent désormais à l'EuroAirport. Avec une desserte ferroviaire sans arrêt et un bon contournement de Mulhouse par la Gare du Nord (Figure 7), la capitale de l'Europe pourrait être accessible à moins d'une heure du 5 e aéroport français de province (6,5 millions de passagers en 2014) alors que le sien stagne autour du million de passagers. Du coup, le deuxième scénario est lui aussi remis en selle, avec un rapprochement entre Dijon et Besançon, des collaborations entre le Nord-est FrancheComté et le Jura suisse...

\section{Conclusion}

La région Rhin-Rhône cherche son chemin depuis des décennies (Gibert, 1930 ; Dézert, 1969 ; Woessner, 2000). Axe de transit relativement peu fréquenté, l'autoroute A 36 n'a pas généré de système territorial. Le canal à grand gabarit est mort-né en 1997. L'industrie est davantage préoccupée par la réduction du nombre de ses établissements et de ses salariés que d'une stratégie offensive. Les services publics et privés universités, services aux entreprises, culture, tourisme, ingénierie de projets sociaux ne nouent guère de partenariats et de métissages au contact des mondes latin et rhénan.

51 La LGV de 2011 aurait pu être le kairos, le petit moment extraordinaire des Grecs, lorsque dans le cours de l'histoire s'annonce une opportunité que l'on ne peut saisir qu'une fois. La gouvernance à la française ne l'a pas permis. Deux ans après la création de la MRR, le 20 octobre 2009, le discours présidentiel de Saint-Dizier annonçait la fin des Contrats métropolitains au profit des Pôles métropolitains; ainsi, versatile, l'Etat ne laisse pas le temps à des projets d'aménagement de se mettre en ordre de bataille. Car il faut des années sinon plusieurs décennies pour qu'un système territorial puisse monter en puissance. Dans une logique de gouvernance rhénane, cela se serait passé tout autrement : la pratique de la subsidiarité permet aux entités de base de proposer des projets selon une logique bottom up, du bas vers le haut. Or, les collectivités de la MRR avaient bel et bien exprimé le désir de se fédérer autour de la LGV.

Néanmoins, il se passe quelque chose de nouveau dans le monde de la grande vitesse ferroviaire où l'on voit que Rhin-Rhône est au contact de deux univers, celui de la France et celui du Rhin. La grande vitesse à la française, ce sont des liaisons entre Paris et les métropoles de province, sans arrêt; le tronçon Paris-Dijon illustre cette conception. La grande vitesse rhénane s'arrête plus souvent et elle dessert des villes plus modestes; ce qu'on retrouve entre Dijon et Mulhouse où le TGV devient rhénan, avec des arrêts parfois toutes les 20 minutes à Dijon, Besançon, Belfort-Montbéliard, 
Mulhouse et Bâle. On voit ainsi s'opposer l'univers centralisé à la française jusqu'à Dijon, et au-delà, les armatures urbaines polynucléaires du monde rhénan. De quoi avoir le vertige à propos des permanences historiques quand on superpose la ligne du TGV Rhin-Rhône au tracé de la frontière du Royaume de France et de l'empire de Charles-Quint (Figure 10). On a donc une zone indécise de contact. Deux systèmes se rencontrent, incarnés d'un côté par la SNCF et RFF, et de l'autre par la Deutsche Bahn et les CFF. Ils vont nécessairement se faire concurrence mais devraient aussi apprendre l'un de l'autre. "Le temps heureux du roi d'Espagne », comme on l'entendait en Franche-Comté, avec une administration lointaine et bienveillante, peut-il revenir? L'Union européenne est bien dans ce concept avec la subsidiarité...

Figure 10 : Quand le TGV Rhin-Rhône révèle l'histoire

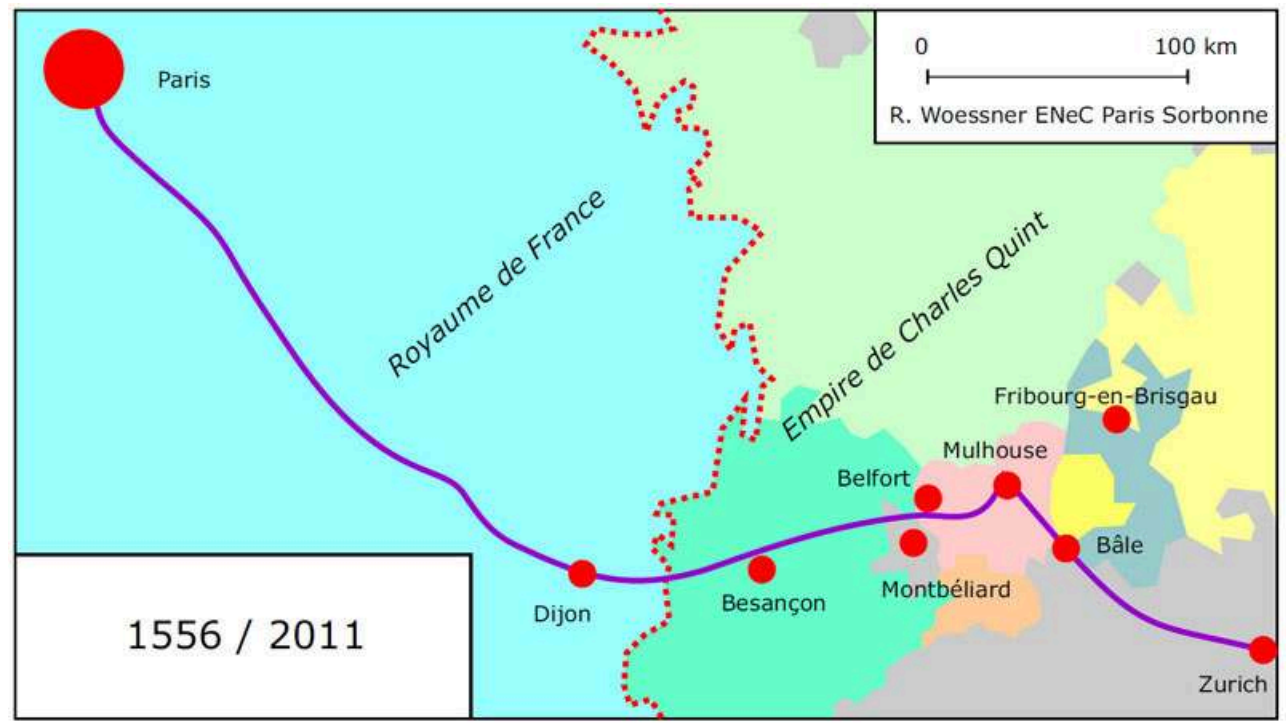

\section{BIBLIOGRAPHY}

APUR, 2009, Le système ferroviaire Lyon-Austerlitz : une gare à Bercy ?, Document d'étude, 59 p.

Bavoux J.-J., 1993, Le « carrefour » bourguignon, analyse d'un espace de circulation, Thèse de doctorat, Dijon, 3 tomes, $945 \mathrm{p}$.

Bérion P., Duféal M., 2006, Structuration territoriale de la Franche-Comté. Essai d'identification et de hiérarchisation des bourgs, petites villes et pôles urbains, Rapport final Alpcity, 125 p.

DATAR, 1994, Débat national pour l'aménagement du territoire, Paris La Documentation française, 75 p.

Dézert B., 1969, La croissance industrielle et urbaine de la Porte d'Alsace, Thèse de géographie, Paris SEDES, $520 \mathrm{p}$. 
Gibert A., 1930, La Porte de Bourgogne et d'Alsace, Thèse de géographie, Besançon, Jacques et Demontrond.

Mignot J.-L., 2011, L'arrivée de la grande vitesse ferroviaire dans l'Est de la France : un espoir de désenclavement de la Haute-Saône par un renouveau des axes méridiens?, Thèse de géographie, Université de Nancy 2, 498 p.

Obifive, 2007, Métropole Rhin-Rhône, projet métropolitain, Paris 63 p.

Offner J.-M., 1993, « Les « effets structurants » du transport : mythe politique, mystification scientifique », L'Espace géographique $\mathrm{N}^{\circ} 3$, p. 233-242.

Richer C., Bérion P., 2010, « Le rôle des grandes infrastructures de transport dans la structuration des espaces régionaux : le cas de l'arrivée du TGV dans le réseau métropolitain Rhin-Rhône », Colloque Villes et grands équipements de transport, Belgeo 2010-1-2, Bruxelles 242 p., cf. p. 159-169.

Woessner R., 2000, Mythe et réalité de l'espace Rhin-Rhône, La dynamique industrielle comme facteur de recomposition territoriale, Thèse de géographie, Presses Universitaires Franc-Comtoises, Besançon 2000, 360 p.

Woessner R., 2005, « L'éternel retour du Corridor Rhin-Rhône », Annales de Géographie n647, janvier-février 2006, Paris A. Colin, p. 2 à 25.

Woessner R., 2008, La Métropole Rhin-Rhône : vers l'émergence d'un territoire ? Editions Jérôme Do Bentzinger, Colmar, $253 \mathrm{p}$.

Woessner R., 2011, La Métropole Rhin-Rhône : mission impossible ou émergence d'un territoire, Colloque Transports, territoires et société, UTBM-UHA, Collection Histoire industrielle et société, Picard, 275 p., cf. 219-231.

\section{NOTES}

1. La SACM de Belfort devient Alsthom en 1928. Le groupe s'intitule GEC-Alsthom en 1989, enfin Alstom en 1998.

2. http://ass-tgv-rhin-rhone.net, un site qui a disparu en 2012.

3. Selon les lignes TER, les correspondances avec les TGV sont réussies (Strasbourg-Bâle) ou ratées (depuis Altkirch et Belfort, dans la plupart des cas, il faut tabler sur un temps d'attente de 45 à 60 minutes en gare de Mulhouse).

4. L'Alsace, 21 février 2012.

5. http://www.besancon.fr/index.php?p=1024\&art_id=3701\#LGV\%20paysage\%20eco, janvier 2012.

6. Depuis l'introduction du péage à la Gare Picardie, celle de Montchanin est la seule gare TGV française disposant d'un parking gratuit.

7. http://www.lajonxion.fr/, janvier 2012.

8. Observatoire des gares du TGV Rhin-Rhône, janvier 2009, Besançon Théma.

9. M. Pribetich, Séminaire interacadémique, Dijon CRDP, 22 et 23 mars 2005. 


\section{ABSTRACTS}

Appearing in 2011, the Rhin-Rhône TGV slips into the interstice left by the South-East and East TGV. It serves territories weakened by the decline of the manufacturing industry. Considering the complexity of the problems, the strategy of the actors and the intricacy of geographical scales, eight pragmatic questions will be asked in order to try to understand what is tied from now on: Is the Rhin-Rhône high speed line the result of a local lobbying? Has the Trans Europe Rhin-Rhône Méditerranée Association reached its target? Is the Rhin-Rhône TGV really necessary? What are the dangers threatening the achievement of the LGV? Are the people in Dijon mistaken when they plan two stations? Is the Mulhouse station a center of growth? Are the new stations condemned to be in the middle of nowhere? Is the Métropole RhinRhône a stillborn concept?

Apparu en 2011, le TGV Rhin-Rhône se glisse dans un interstice laissé par les TGV Sud-Est et Est. Il dessert des territoires fragilisés par le déclin de l'industrie manufacturière. Face à la complexité des problèmes, du jeu des acteurs et de l'enchevêtrement des échelles géographiques, on se posera huit questions pragmatiques pour essayer de comprendre ce qui se noue désormais : La LGV Rhin-Rhône est-elle le produit d'un lobbying local? L'objectif de l'Association Trans Europe Rhin-Rhône Méditerranée est-il atteint ? Le TGV Rhin-Rhône est-il nécessaire ? Quels sont les périls menaçant l'achèvement de la LGV? Avec un projet comportant deux gares, les Dijonnais sont-ils dans l'erreur? La gare de Mulhouse est-elle un pôle de croissance? Le syndrome des betteraves menace-t-il les gares nouvelles? La Métropole Rhin-Rhône est-il un concept mort-né ?

Der TGV Konzept ist gründlich für eine rasche Verbindung zwischen Paris und die anderen französischen oder europaischen Grossstädte ausgedacht. Also müssen die kleine und die mittlere Städte sich eilen, wenn sie eine TGV Linie, einen TGV Bahnhof und stoppende Züge erhalten wollen. Das alles in acht fragen für den TGV Rhin-Rhône: Ist der Bau der Hochgeschwingdigskeitlinie eine Konsequenz einer Lobbyingpolitik? Hat der lokaler Verband Trans Europe Rhin-Rhône Méditerranée seine Ziele erreicht ? Ist eigentlich der TGV Rhin-Rhône notwendig? Welche Gefahre drohen die Befertigung der Linie? Würden die zwei geplänte Bahnhöfe in Dijon ein Irrtum sein? Kann der Mülhauser Bahnhof ein Wächstummagnet sein? Selbe Frage für die neuen Bahnhöfen, die mann aüsser der Städte gebaut hat? Ist die Metropolregion Rhin-Rhone ein Plan, der von vornherein zum scheitern verurteilt ist?

\section{INDEX}

Schlüsselwörter: Hochgeschwingdigskeitzug, neuer Bahnhof, Rhein-Rhône, Territorialisierung, zentraler Ort, Zwischenraum

Mots-clés: gares nouvelles, grande vitesse ferroviaire, lieu central, Rhin-Rhône, territoire de marge, territorialisation

Keywords: central place, High Speed Railway, interstitial space, new station, Rhine-Rhone, territorialization 
AUTHOR

\section{RAYMOND WOESSNER}

Paris Sorbonne, Laboratoire Espace, Nature et Culture, UMR 8185 - Institut de Géographie, 191

rue Saint-Jacques, 75005 Paris - raymond.woessner@wanadoo.fr 